\title{
K-10 and KSF Clays as Green and Recyclable Heterogeneous Catalysts for the Cannizzaro Reaction Using DABCO under MWI and Solvent-free Conditions
}

\author{
OMID MARVI* and MARYAM TALAKOUBI \\ 'Department of Chemistry, Payam Noor University, PO Box 19395-3697 Tehran, Iran. \\ ${ }^{*}$ Corresponding Author E-mail: omid_marvi@pnu.ac.ir \\ http://dx.doi.org/10.13005/ojc/320140
}

Received: January 11, 2016; Accepted: February 26, 2016

\begin{abstract}
Montmorillonite K-10 and KSF clays as recyclable and heterogeneous catalysts are used to catalyze the Cannizzaro reaction by 1,4-diazabicyclo[2.2.2]octane (DABCO) under microwave irradiation (MWI) and solvent-free conditions in excellent yields within seconds. The solid clays applied in the first cycle are recovered and reused in the subsequent reactions.
\end{abstract}

Keywords: Cannizzaro; DABCO; MWI ; KSF and K-10 clays

\section{INTRODUCTION}

The redox disproportionative conversion of aldehydes into their corresponding alcohols and carboxylic acids, known as Cannizzaro reaction ${ }^{1}$,is one of the oldest processes in synthetic organic chemistry and is classically conducted at elevated temperatures using equimolar or excessive amounts of alkali metal hydroxides or other strong bases. Such harsh conditions and competitive formation of undesired side products have been the main limitations for Cannizzaro reaction in the past several decades. ${ }^{2}$ Many developments have been made to the original version of the reaction in the past several years by means of microwave irradiation, ${ }^{3}$ ultrasound mediation, ${ }^{4}$ cation templates, ${ }^{5}$ solid-supported reagents, ${ }^{6}$ solvent-free conditions, ${ }^{7}$ crossed Cannizzaro version of the reaction, ${ }^{3 b, 6}$ gas-phase process, ${ }^{8}$ and Lewis acid catalysis. ${ }^{9}$ In a few examples conducted at room temperature, strong basic media are still required ${ }^{7,10}$ or yields of the majority of the reactions are rather low or moderate. ${ }^{9 c}$ Despite all of these modifications, the reaction still requires drastic conditions in most cases.

Natural alumosilicates, such as zeolites and clays, are solid acids that could act as an efficient alternative to liquid acids. Natural and modified clays have attracted attention due to their extremely versatile properties and high potential in green chemistry. Though the physicochemical properties of the clays are similar their BET surface areas differ. $\mathrm{K}-10$ Has a higher surface area (about $250 \mathrm{~m}^{2} / \mathrm{g}^{1}$ ) compared to that of $\operatorname{KSF}\left(10 \mathrm{~m}^{2} / \mathrm{g}^{1}\right) \cdot{ }^{11}$ 
The combined use of montmorillonites and microwave irradiation (MWI) showed rate enhancements, high yields and short reaction times. In addition, the use of solvent- free conditions with heterogeneous media under microwave irradiation is a useful alternative and has received considerable attention due to its greater efficiency from economic as well as ecological points of view. ${ }^{12}$

Recently, use of nonmetallic reagents is an area of growing interest because of environmental regulations. Among the various organic bases,1,4diazabicyclo[2,2,2]octane (DABCO) has been employed as an efficient organic base to bring about various organic transformations. ${ }^{13-15}$

In continuation of ongoing investigations exploring the use of montmorillonites as solid supports in the synthesis and reactivity of organic compounds using microwave irradiation, ${ }^{16}$ this work reports a simple, green and environmentally acceptable microwave promoted the Cannizzaro reaction by DABCO over $\mathrm{K}-10$ and KSF clays as efficient heterogeneous catalysts (Scheme 1). The solid clays can be recovered later and reused in the subsequent reactions. Details of recyclability of the recovered clays have been shown in the Table 1.

Results summarized in the Table indicate the scope and generality of the reaction with respect to the various benzaldehydes. The nature of the substituents on the aromatic ring of benzaldehyde has different influences. The presence of the electron withdrawing groups such as nitro and fluorine which increase the polarity of the carbonyl group inductively, give high yields of products as well as the short reaction time compared to the electron donating groups like methyl and methoxy. On the other hand, the percentage of the products obtained by the $\mathrm{K}-10$ clay is more than those prepared by the KSF clay. This observation can be attributed to the more surface area in K-10.
The mechanism of this reaction is well known and is described as below (Scheme 2). Here, we only emphasize minor differences and advantages that are due to the use of solid catalysis. As this reaction is carried out in a solvent-free, open reaction system, the key step is the adsorption of the carbonyl compound on the surface of the catalyst. Montmorillonite is considered to be a mixed Brønsted-Lewis solid acid. The majority of the acid centers are of Lewis acid type. ${ }^{17}$ The adsorption occurs when the electron rich carbonyl oxygen interacts with a surface Lewis acid center, and it is of rather chemical than physical nature. This Lewis acid-base interaction anchors the aldehyde and initiates strong electrophilic character on the carbonyl carbon, resulting in a surface-bound intermediate of carbocationic nature.

\section{EXPERIMENTAL}

Chemicals were purchased from Aldrich and Merck chemical companies and used without further purification. Montmorillonite KSF clay, surface area $15 \pm 10 \mathrm{~m}^{2} / \mathrm{g}$, chemical composition (average value): $\mathrm{SiO}_{2}(54.0 \%), \mathrm{Al}_{2} \mathrm{O}_{3}(17.0 \%), \mathrm{Fe}_{2} \mathrm{O}_{3}(5.2 \%)$, $\mathrm{CaO}(1.5 \%), \mathrm{MgO}(2.5 \%), \mathrm{Na}_{2} \mathrm{O}(0.4 \%), \mathrm{K}_{2} \mathrm{O}(1.5 \%)$ and montmorillonite K10 clay,surface area: $(200 \pm 10)$ $\mathrm{m}^{2} / \mathrm{g}$, chemical composition (average value): $\mathrm{SiO}_{2}$ (73.0\%), $\mathrm{Al}_{2} \mathrm{O}_{3}(14.0 \%), \mathrm{Fe}_{2} \mathrm{O}_{3}(2.7 \%), \mathrm{CaO}(0.2 \%)$, $\mathrm{MgO}(1.1 \%), \mathrm{Na}_{2} \mathrm{O}(0.6 \%), \mathrm{K}_{2} \mathrm{O}(1.9 \%)$ were purchased from Fluka chemical company. Melting points were measured on an Electro thermal 9100 apparatus. ${ }^{1} \mathrm{H}$ NMR and ${ }^{13} \mathrm{CNMR}$ spectra were recorded by a FTNMR BRUKER DRX 500 Avence spectrometer $\left(500 \mathrm{MHz}\right.$ for ${ }^{1} \mathrm{H}$ NMR and $125 \mathrm{MHz}$ for ${ }^{13} \mathrm{C}$ NMR). Chemical shifts were measured in ppm from TMS. DMSO- $d_{6}$ was used as solvent as well as the internal standard. Physical and spectral data of the synthesized compounds are given in the Supplementary material to this paper. Microwave irradiations were realized with a Synthewave $402^{\circledR}$

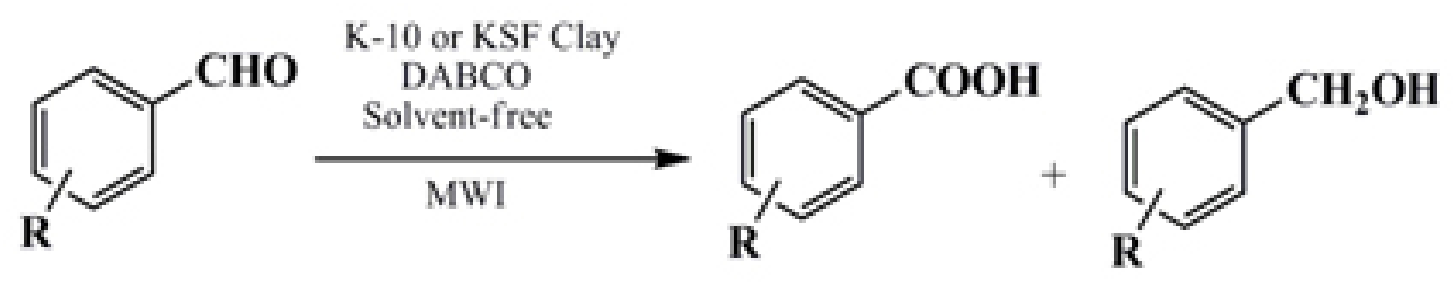

Scheme 1: Solvent-free Cannizzaro reaction by DABCO on KSF and K-10 clays and MWI. 
Table 1: The yields and reaction times for the solvent-free Cannizzaro reaction by $\mathrm{DABCO}$ on $\mathrm{KSF}$ and $\mathrm{K}-10$ clays under $\mathrm{MWI}$

\begin{tabular}{|c|c|c|c|c|}
\hline Entry & Aldehyde & Acid $^{a}$ & $\begin{array}{l}\text { Yield (\%) } \\
\text { K10/KSF }\end{array}$ & Time (s) \\
\hline 1 & & & 95(91)/92(89) & 30 \\
\hline 2 & & & $94(92) / 92(88)$ & 30 \\
\hline 3 & & & $96(93) / 94(90)$ & 15 \\
\hline 4 & & & $98(94) / 94(89)$ & 20 \\
\hline 5 & & & $98(96) / 95(90)$ & 15 \\
\hline 6 & & & $91(88) / 90(85)$ & 40 \\
\hline 7 & & & $97(94) / 94(90)$ & 20 \\
\hline 8 & & & $94(90) / 91(86)$ & 30 \\
\hline 9 & & & $88(84) / 87(82)$ & 40 \\
\hline 10 & & & $95(92) / 92(86)$ & 30 \\
\hline 11 & & & $96(85) / 91(87)$ & 15 \\
\hline 12 & & & $96(93) / 94(90)$ & 20 \\
\hline
\end{tabular}

a All the benzoic acid derivatives are well known compounds (literature and/ or commercial sources).

b Yields are based on isolated acids. Yield of alcohols was almost the same as acid in each case. Yields indicated in the parenthesis correspond to those reactions which the recovered clays were used. The fresh solid clay portion applied in the first cycle was filtered off at the end of reaction, washed with methanol $(2 \times 30 \mathrm{~mL})$ and dried at $120^{\circ} \mathrm{C}$ under the reduced pressure to be reused in the subsequent reaction. 
(Prolabo, France) single mode focused microwave reactor. ${ }^{18}$

\section{General Procedure}

In a $50 \mathrm{~mL}$ beaker, aldehyde $(1 \mathrm{mmol})$ was roughly mixed with $1 \mathrm{~g}$ montmorillonite. After 2 min of mechanical stirring, DABCO $(10 \mathrm{mmol})$ was added and then mixture was submitted into a single mode focused microwave reactor with continuous rotation for the time described in Table 1 (optimized time) at $40{ }^{\circ} \mathrm{C}$. After completing the reaction (TLC), water $(30 \mathrm{~mL})$ was added to the mixture, the product was washed by $\mathrm{CH}_{2} \mathrm{Cl}_{2}(2 \times 10 \mathrm{~mL})$, clay was filtered, and the solvent was evaporated to give the alcohol component. To obtain the acid component, the filtrate was acidified, extracted with $\mathrm{CH}_{2} \mathrm{Cl}_{2}$, and dried over magnesium sulphate. Then the solvent was evaporated to givethe acid component.The solid clay portion was washed with methanol and dried at $120{ }^{\circ} \mathrm{C}$ under a reduced pressure to be reused in the subsequent reactions which showed the gradual decrease in the activity (Table 1). The filtrate was dried over $\mathrm{MgSO}_{4}$, the organic solvent was evaporated, and aryl alcohol was obtained. Isolated products were characterized by melting points, ${ }^{1} \mathrm{HNMR}$ and ${ }^{13} \mathrm{C}$ NMR spectrometric data and were compared with the literature and/or with authentic samples.

\section{Spectroscopic and analytical data Benzoic acid (entry 1)}

m.p. $120-121^{\circ} \mathrm{C} ;{ }^{1} \mathrm{H}$ NMR $(500 \mathrm{MHz}$, DMSO$\left.d_{6}, \delta / \mathrm{ppm}\right): 8.15(2 \mathrm{H}, d d, J=8.1,1.3 \mathrm{~Hz}$, ortho), $7.49(2 \mathrm{H}, t, J=7.2 \mathrm{~Hz}$, meta) $7.38(1 \mathrm{H}, t, J=8.1$ $\mathrm{Hz}$, para); ${ }^{13} \mathrm{C}$ NMR (125MHz, DMSO- $\left.d_{6}, \delta / p p m\right):$ $172.54(\mathrm{COOH}), 134.76\left(\mathrm{C}_{4}\right), 133.42\left(\mathrm{C}_{2}\right), 131.26$ $\left(C_{1}\right), 129.77\left(C_{3}\right)$.

\section{4-Bromobenzoic acid (entry 2)}

m.p. $151-152^{\circ} \mathrm{C} ;{ }^{1} \mathrm{H} \mathrm{NMR}(500 \mathrm{MHz}$, DMSO$\left.d_{6}, \delta / \mathrm{ppm}\right): 7.91(2 \mathrm{H}, d, J=8.2 \mathrm{~Hz}$, aromatic), $7.48\left(2 \mathrm{H}, d, J=8.4 \mathrm{~Hz}\right.$, aromatic) $;^{13} \mathrm{C} \mathrm{NMR}(125 \mathrm{MHz}$, DMSO- $\left.d_{6}, \delta / p p m\right): 167.54(\mathrm{COOH}), 131.09\left(\mathrm{C}_{2}\right)$, $130.49\left(\mathrm{C}_{3}\right), 129.65(\underline{\mathrm{CCOOH}}), 127.43(\mathrm{CBr})$.

\section{4-Fluorobenzoic acid(entry 3)}

m.p. $184-186^{\circ} \mathrm{C} ;{ }^{1} \mathrm{H} \mathrm{NMR}(500 \mathrm{MHz}$, DMSO$\left.d_{6}, \delta / \mathrm{ppm}\right): 8.19\left(2 \mathrm{H}, t,{ }^{3} \mathrm{~J}_{\mathrm{H}-\mathrm{H}}=7.8 \mathrm{~Hz}\right.$, aromatic), $7.11\left(2 \mathrm{H}, d,{ }^{2} J_{\mathrm{H}-\mathrm{H}}=8.2 \mathrm{~Hz}\right.$, aromatic) $;{ }^{13} \mathrm{C} \mathrm{NMR}$ (125MHz, DMSO- $\left.d_{6}, \delta / p p m\right): 171.12(\mathrm{COOH}), 167.15$ $\left(d,{ }^{1} J_{\mathrm{C}-\mathrm{F}}=254.2 \mathrm{~Hz}\right), 132.47\left(d,{ }^{3} J_{\mathrm{C}-\mathrm{F}}=9.6 \mathrm{~Hz}\right), 125.54$ $\left(d,{ }^{4} J_{C-F}=3.4 \mathrm{~Hz}\right), 115.63\left(d,{ }^{2} J_{C-F}=22.35 \mathrm{~Hz}\right)$.

\section{3-Nitrobenzoic acid (entry 4)}

m.p. $140-143^{\circ} \mathrm{C} ;{ }^{1} \mathrm{H}$ NMR $(500 \mathrm{MHz}$, DMSO$\left.d_{6}, \delta / \mathrm{ppm}\right): 8.11(\mathrm{~s}, 1 \mathrm{H}), 8.02(d, J=7.7 \mathrm{~Hz}, 1 \mathrm{H}), 7.98$ $(d, J=7.8 \mathrm{~Hz}, 1 \mathrm{H}), 7.65(d, J=7.8 \mathrm{~Hz}, 1 \mathrm{H}), 7.59(t, J$ $=7.7 \mathrm{~Hz}, 1 \mathrm{H})$ ppm. ${ }^{13} \mathrm{CNMR}\left(125 \mathrm{MHz}\right.$, DMSO- $d_{6}, \delta /$ ppm):174.21(COOH), $137.57\left(\mathrm{CNO}_{2}\right), 131.96\left(\mathrm{C}_{4}\right)$, $131.29(\underline{\mathrm{CCOOH}}), 129.76\left(\mathrm{C}_{6}\right), 129.98\left(\mathrm{C}_{3}\right), 128.28$ $\left(\mathrm{C}_{5}\right) \mathrm{ppm}$.

\section{4-Nitrobenzoic acid (entry 5)}

m.p. $239-243{ }^{\circ} \mathrm{C} ;{ }^{1} \mathrm{H}$ NMR $(500 \mathrm{MHz}$, DMSO- $\left.d_{6}, \delta / \mathrm{ppm}\right): 8.12(2 \mathrm{H}, d, J=8.1 \mathrm{~Hz}$, aromatic), $7.65\left(2 \mathrm{H}, d, J=8.1 \mathrm{~Hz}\right.$, aromatic): ${ }^{13} \mathrm{C} N \mathrm{NMR}(125 \mathrm{MHz}$, DMSO- $\left.d_{6}, \delta / p p m\right): 169.21(\mathrm{COOH}), 141.13\left(\mathrm{CNO}_{2}\right)$, $131.54\left(\mathrm{C}_{2}\right), 129.18(\underline{\mathrm{CCOOH}}), 128.34\left(\mathrm{C}_{3}\right)$.

\section{4-Methylbenzoic acid (entry 6)}

m.p. $181-184{ }^{\circ} \mathrm{C} ;{ }^{1} \mathrm{H}$ NMR $(500 \mathrm{MHz}$, DMSO$\left.d_{6}, \delta / \mathrm{ppm}\right): 8.18(2 \mathrm{H}, d, J=8.2 \mathrm{~Hz}$, aromatic $), 7.23$ $\left(2 \mathrm{H}, d, J=8.5 \mathrm{~Hz}\right.$, aromatic), $2.54\left(3 \mathrm{H}, s, \mathrm{CH}_{3}\right) ;{ }^{13} \mathrm{C}$ NMR (125MHz, DMSO- $\left.d_{6}, \delta / p p m\right): 175.13(\mathrm{COOH})$, $152.95\left(\underline{\mathrm{CCH}}_{3}\right), 134.36\left(\mathrm{C}_{2}\right), 123.47(\underline{\mathrm{CCOOH}})$, $121.56(\underline{\mathrm{CCOCH}}), 31.92\left(\mathrm{CH}_{3}\right)$.

\section{4-Nitrobenzoic acid (entry 7)}

m.p. $142-145^{\circ} \mathrm{C} ;{ }^{1} \mathrm{H}$ NMR $(500 \mathrm{MHz}$, DMSO$\left.d_{6}, \delta / \mathrm{ppm}\right): 8.12(1 \mathrm{H}, d, J=8.0 \mathrm{~Hz}, \mathrm{CHCCOOH})$, 7.49-7.41 $(2 \mathrm{H}, m$, aromatic) $7.39-7.22(1 \mathrm{H}$, $\left.m, \mathrm{CNO}_{2} \mathrm{C} \underline{\mathrm{H}}\right) ;{ }^{13} \mathrm{CNMR}\left(125 \mathrm{MHz}\right.$, DMSO- $d_{6}, \delta /$ ppm):176.59(COOH), $142.83\left(\mathrm{CNO}_{2}\right), 135.48\left(\mathrm{C}_{4}\right)$, $132.77(\mathrm{CCOOH}), 131.91\left(\mathrm{C}_{6}\right), 129.18\left(\mathrm{C}_{3}\right), 127.86$ $\left(C_{5}\right)$.

\section{3-Chlorobenzoic acid (entry 8)}

m.p. 151-155 ${ }^{\circ} \mathrm{C} ;{ }^{1} \mathrm{H}$ NMR $(500 \mathrm{MHz}$, DMSO$\left.d_{6}, \delta / \mathrm{ppm}\right): 8.09(\mathrm{~s}, 1 \mathrm{H}), 7.93(d, J=7.8 \mathrm{~Hz}, 1 \mathrm{H}), 7.96$ $(d, J=7.8 \mathrm{~Hz}, 1 \mathrm{H}), 7.59(d, J=7.9 \mathrm{~Hz}, 1 \mathrm{H}), 7.43(t$, $J=7.7 \mathrm{~Hz}, 1 \mathrm{H})$ ppm. ${ }^{13} \mathrm{CNMR}\left(125 \mathrm{MHz}\right.$, DMSO- $d_{6}$, $\delta / p p m): 173.45(\mathrm{COOH}), 135.21(\mathrm{CCl}), 131.96\left(\mathrm{C}_{4}\right)$, $130.48(\underline{\mathrm{CCOOH}}), 129.94\left(\mathrm{C}_{6}\right), 129.73\left(\mathrm{C}_{3}\right), 128.62$ $\left(\mathrm{C}_{5}\right) \mathrm{ppm}$.

\section{4-Methoxybenzoic acid (entry 9)}

m.p. $181-183^{\circ} \mathrm{C} ;{ }^{1} \mathrm{H}$ NMR(500MHz, DMSO$\left.d_{6}, \delta / \mathrm{ppm}\right): 8.02(2 \mathrm{H}, d, J=8.5 \mathrm{~Hz}$, aromatic), 7.11 $\left(2 \mathrm{H}, d, J=8.6 \mathrm{~Hz}\right.$, aromatic), $3.78\left(3 \mathrm{H}, s, \mathrm{CH}_{3}\right) ;{ }^{13} \mathrm{C}$ NMR (125MHz, DMSO- $\left.d_{6}, \delta / p p m\right): 171.55(\mathrm{COOH})$, 

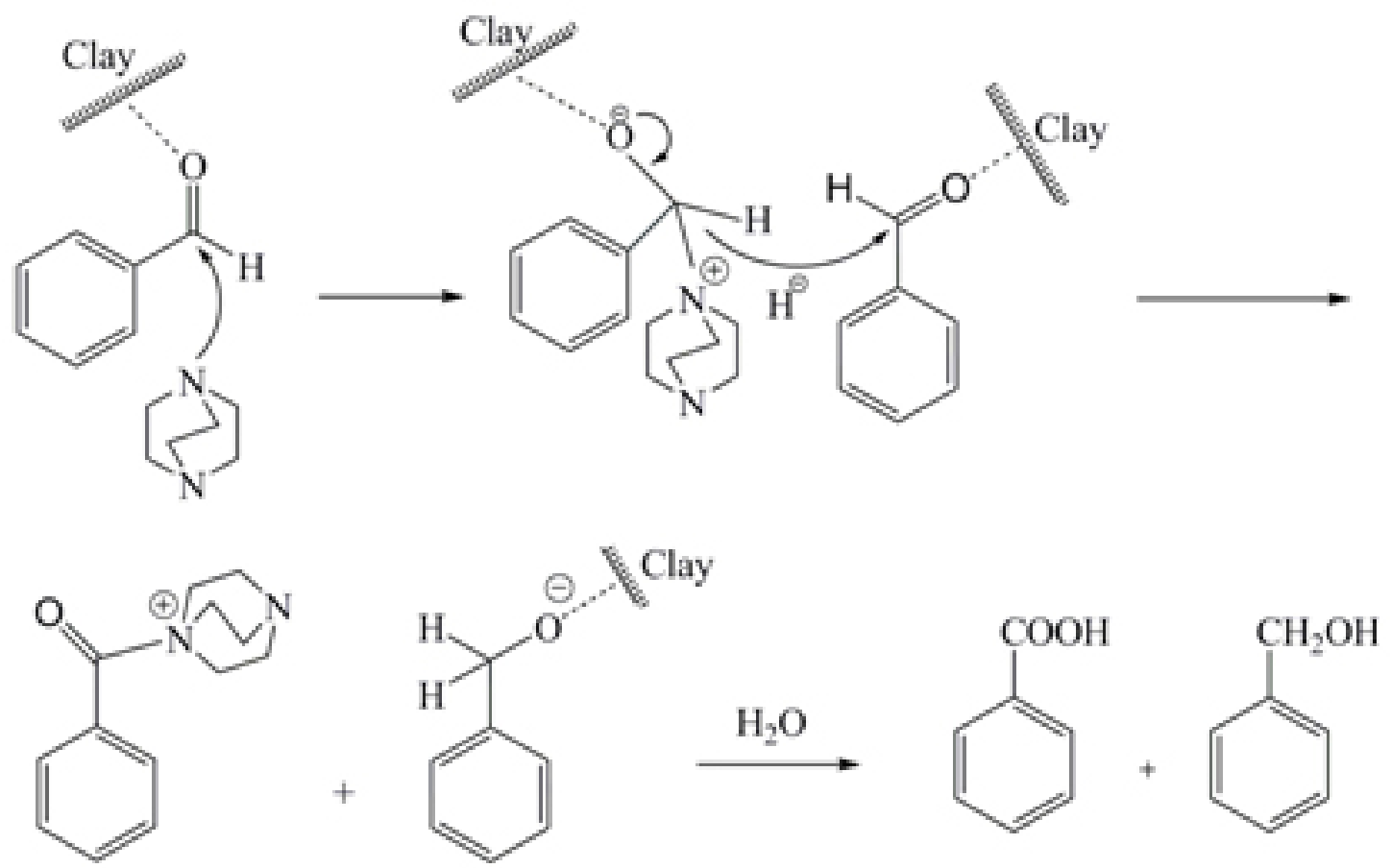

Scheme 2: Possible mechanism for Cannizzaro reactionby DABCO over clay under MWI.

$163.67\left(\underline{\mathrm{COCH}_{3}}\right), 133.72\left(\mathrm{C}_{2}\right), 121.54(\underline{\mathrm{CCOOH}})$, $114.57\left(\mathrm{CCOCH}_{3}\right), 55.38\left(\mathrm{CH}_{3}\right)$.

\section{4-Chlorobenzoic acid(entry 10)}

m.p. $139-142{ }^{\circ} \mathrm{C} ;{ }^{1} \mathrm{H}$ NMR $(500 \mathrm{MHz}$, DMSO- $\left.d_{6}, \delta / \mathrm{ppm}\right): 8.07(1 \mathrm{H}, d, J=8.2 \mathrm{~Hz}$, $\mathrm{C}$ HCCOOH), 7.42-7.38 (2H, $m$, aromatic) $7.35-7.29$ $(1 \mathrm{H}, m, \mathrm{CCICH}) ;{ }^{13} \mathrm{CNMR}\left(125 \mathrm{MHz}, \mathrm{DMSO}-d_{6}, \delta /\right.$ ppm):172.21(COOH), $135.93(\mathrm{CCl}), 133.59\left(\mathrm{C}_{4}\right)$, $132.28(\mathrm{CCOOH}), 131.75\left(\mathrm{C}_{6}\right), 128.43\left(\mathrm{C}_{3}\right), 126.81$ $\left(\mathrm{C}_{5}\right)$.

\section{2-Fluorobenzoic acid (entry 11)}

m.p. $123-124{ }^{\circ} \mathrm{C} ;{ }^{1} \mathrm{H}$ NMR $(500 \mathrm{MHz}$, DMSO- $\left.d_{6}, \delta / \mathrm{ppm}\right): 8.09(1 \mathrm{H}, d, J=8.0 \mathrm{~Hz}$, $\mathrm{CHCCOOH}), 7.55(1 \mathrm{H}, m, \mathrm{CFCH}), 7.28-7.19(2 \mathrm{H}$, $m$, aromatic); ${ }^{13} \mathrm{CNMR}\left(125 \mathrm{MHz}\right.$, DMSO- $\left.d_{6}, \delta / \mathrm{ppm}\right)$ : 170.12(COOH), $163.65\left(d,{ }^{1} J_{\mathrm{C}-\mathrm{F}}=261.2 \mathrm{~Hz}\right), 134.72$ $\left(d,{ }^{3} J_{C-F}=9.4 \mathrm{~Hz}\right), 131.67\left(\mathrm{C}_{6}\right), 124.38\left(d,{ }^{3} \mathrm{~J}_{\mathrm{C}-\mathrm{F}}=2.5\right.$ $\mathrm{Hz}), 117.53\left(d,{ }^{2} J_{\mathrm{C}-\mathrm{F}}=10.28 \mathrm{~Hz}\right), 117.12\left(d,{ }^{2} J_{\mathrm{C}-\mathrm{F}}=\right.$ $21.98 \mathrm{~Hz})$.

\section{2-Chlorobenzoic acid (entry 12)}

m.p. $238-2400^{\circ} \mathrm{C} ;{ }^{1} \mathrm{H} \mathrm{NMR}(500 \mathrm{MHz}$, DMSO$\left.d_{6}, \delta / \mathrm{ppm}\right): 8.08(2 \mathrm{H}, d, J=8.2 \mathrm{~Hz}$, aromatic), 7.54 $\left(2 \mathrm{H}, d, J=8.3 \mathrm{~Hz}\right.$, aromatic); ${ }^{13} \mathrm{C}$ NMR $(125 \mathrm{MHz}$, DMSO- $\left.d_{6}, \delta / \mathrm{ppm}\right): 168.08(\mathrm{COOH}), 138.57(\mathrm{CCl})$, $130.49\left(\mathrm{C}_{2}\right), 128.65(\underline{\mathrm{COOH}}), 127.81\left(\mathrm{C}_{3}\right)$.

In conclusion, a simple and efficient procedure for the clay catalyzed Cannizzaro reaction in the presence of 1,4-diazabicyclo[2.2.2]octane (DABCO)under MW irradiation and solvent-free conditions was established. This present method is superior since it is eco-friendly, advantageous over previously described methods in yield, requires no special apparatus, there is simplicity of operation, and is non-hazardous, simple and convenient. In addition, the simple experimental and product isolation procedures combined with the easy recovery and reuse of these worthwhile natural clays play an important role in development of the clean and environmentally friendly strategy in this new and benign method. 


\section{REFERENCES}

1. (a) Cannizzaro, S.Ann,1853,88, 129-130. b) Geissman, T. A.Org. React. 1944,2, 94-113.

2. (a) Fuson R. C.; Emerson W. S.; Gray H. W. J. Am. Chem. Soc.1939, 61, 480-482.

(b) Maruyama, K.; Murakami, Y.; Yoda, K.;Mashino,T.; Nishinaga, A.J .Chem. Soc. Chem.Commun.1992, 1617-1618.

(c)Jin, S. J.;Arora, P. K.;Sayre, L. M.J. Org. Chem.1990, 55, 3011-3018.

(d) Okuyama, T.; Kimura, K.;Fueno, T.Bull. Chem. Soc.Jpn.1982, 55, 2285-2286.

3. (a) Sharifi, A.;Mojtahedi, M. M.;Saidi, M. R. Tetrahedron Lett.1999, 40, 1179-1180.

(b) Varma, R. S.;Naicker, K.P.;Liesen, P. J.Tetrahedron Lett.1998, 39, 8437-8440.

4. Entezari,M.H.;Shameli, A. A.Ultrason Sonochem. 2000, 7, 169-172.

5. Vida, Y.; Perez-Inestrosa, E.;Suau, R. Tetrahedron Lett. 2005, 46, 1575-1577.

6. Reddy, B.;Srinivas, V. S.; Yadav,R., J.S.; Ramalingam, T.Synth. Commun. 2002, 32,219-222.

7. Yoshizawa, K.; Toyota, S.;Toda, F. Tetrahedron Lett. 2001, 42,7983-7985.

8. Sheldon, J. C.; Bowie, J. H.;Dua, S.; Smith, J. D.;O'Hair, R. A. J. J. Org. Chem. 1997, 62, 3931-3937.

9. (a) Curini, M.;Epifano, F.;Genovese, S.;Marcotullio, M. C.;Rosati, O.Org.Lett., 2005, 7,1331-1333.

(b) Russell, A .E.; Miller, S. P.; Morken, J. P. J.Org. Chem. 2000, 65, 8381-8383.

(c) Yang, X. G.;Guo, J. P.;Zou, G.Lett.Org. Chem.2005,2, 145-147.

10. (a) Abaee, M. S.;Mojtahedi, M. M.; Zahedi, M. M.Synlett. 2005, 2317-2320.

(b)Abaee, M. S.;Sharifi, R.;Mojtahedi, M. M.Org. Lett. 2005, 7, 5893-5895.

(c) Ishihara, K.; Yano, T.;Fushimi, M.J. Fluorine Chem. 2008, 129, 994-997.

(d) Baelen, G. V. \& Maes, B. U.W., Tetrahedron 2008, 64, 5604-5619.

11. (a) Lasszlo, P. Science,1987, 235, 14731477.

(b) Baghernejad. B. Lett. Org. Chem.2010,7, 255-257.

(c) Agarwal, S.; Ganguli, J. N.J. Mol. Catal. A:
Chem. 2013, 372, 44-50.

(d) Kaur, N.; Kishore, D.J. Chem. Pharm. Res. 2012, 4, 991-1015.

(e) Dintzner, M. R.Synlett.2013,24, 10911092.

12. (a) Lidström, P.; Tierney, J.; Wathey, B.; Westman, J. Tetrahedron 2001, 57, 92259283.

(b) Braibante, M. E. F.; Braibante, H. T. S.; Morel, A. F.; Costa, C. C.; Lima, M. G.J. Braz. Chem.Soc. 2006, 17,184-188.

(c) Lidstrom, P.; Tierney, J.P. MicrowaveAssisted Organic Synthesis, Eds, Blackwell, Oxford, 2005.

(d) Larhed, M.; Olofsson, K. Microwave Methods in Organic Synthesis, Eds, Springer, Berlin, 2006.

(e) Dallinger, D.; Kappe, C. O.Chem. Rev. 2007, 107, 2563-2591.

13 (a) Zorn,C.;Gnad,F.;Salmen,S.;Herpin,T.;Rei ser,O. TetrahedronLett.2001, 42,7079-7081.

(b) DeSouza,R.O.M.A.;VasConcellos,M.L. A.

A. Catal.Commun.2003, 5, 21-24.

(c) Krishna, P. R.; Sekhar, E. R.; Kannan,V. Tetrahedron Lett.2003, 44, 4973-4975.

(d)Kumar, A.; Pawar, S. S. Tetrahedron 2003, 59, 5019-5026.

14 (a)Cecchi,L.; DeSarlo,F.; Machetti, F. Eur.J.Org.Chem. 2006, 4852-4860.

(b) Bu, X.;Jing, H.; Wang,L.;Chang,T.; Jin,L.;Liang,Y.J. Mol.Catal.A: Chem. 2006, 259,121-124.

15. (a) Murthy,S.N.; Madhav,B.; Nageshwar,Y.V.D. Tetrahedron Lett. 2010, 51,5252-5257.

(b) Bigdeli,M. Chin.Chem.Lett. 2010, 21,11801182.

(c) Krishna,P. R.; Prapurna,Y. L. Tetrahedron Lett. 2010, 51, 6507-6510.

16. (a) Habibi, D.; Marvi, O.Chinese. J. Chem. 2008, 26, 522-524.

(b) Habibi, D.; Marvi, O. Catal. Commun. 2007,8, 127-130.

(c) Marvi, O.;Nikpasand. M. Lett. Org. Chem. 2013, 10, 353-357.

(d) Marvi,O.;Fekri, L. Z.; Takhti, M. Russ. J. Gen.Chem. 2014, 84, 1837-1840.

17. (a) Szöllösi, G.; Török, B.; Baranyi, L.; Bartók, 
M.J.Catal. 1998, 179, 619-623.

(b)Balázsik, K.; Török, B.; Szakonyi, G.; Bartók, M. Applied.Catal. A. 1999,182, 5356.

(c) Török, B.; London, G.; Bartók, M.Synlett 2000, 631-632. (d) Landge, S. M.; Schmidt, A.; Outerbridge,V.; Török, B. Synlett 2007,1600-1604.

18. (a) Prolabo, Fr. Pat. 62241/D, 14669 FR, December $23^{\text {th }} 1991$.

(b) Commarmot.R.; Didenot, R.; Gardais, J.F. Rhône-Poulenc/ Prolabo Patent No. 84/03496 - October $27^{\text {th }} 1986$. 